\title{
Infants of mothers with eating disorders show neurobehavioural and cognitive defects
}

\author{
By Dr Jessica K. Edwards
}

Eating disorders can have serious adverse clinical, social and psychological outcomes in affected patients, but whether maternal eating disorders are associated with negative outcomes in newborns is unknown. Now, a study by Manuela Barona and colleagues has assessed neurobiological and cognitive outcomes in newborns and infants born to mothers with eating disorders. Healthy women and those with an active or past eating disorder were recruited to a prospective longitudinal study during the first or second trimesters of pregnancy. Newborns were assessed at 8 days for neurobehavioural dysregulation using the Brazelton Neonatal Behavioural Assessment Scale, and later assessed at 1 year for cognitive development using the Bayley Scales of Infant and Toddler Development. Newborns from mothers with an active eating disorder showed worse autonomic stability (e.g. poor regulation of breathing or temperature), compared to those from healthy controls. The same trend (although not significant) was identified in newborns of mothers with a past eating disorder. Infants of mothers with a past eating disorder also exhibited delayed language skills and poorer motor development at 1 year compared to healthy controls. The researchers suggest that autonomic stability may be an early biomarker of the effects of maternal eating disorders on offspring, and dysregulated cognitive development at 1 year may be a stable marker of eating disorder risk.

Barona, M., Taborelli, E., Corfield, F., Pawlby, S., Easter, A., Schmidt, U., Treasure, J. \& Micali, N. (2017), Neurobehavioural and cognitive development in infants born to mothers with eating disorders. J Child Psychol Psychiatr. 58: 931-938. doi:10.1111/jcpp.12736

Glossary:

Eating disorder: an unhealthy attitude towards food and often weight/ body image that has adverse medical and psychological consequences; eating disorders can be categorized as either anorexia nervosa, bulimia nervosa, binge eating disorder (BED) or other specified feeding of eating disorder (OSFED)

Prospective longitudinal study: a study that looks for outcomes (e.g. disease development) in the same subjects over a period of time ranging from months to decades. 